\title{
Espondilite tuberculosa em paciente com doença de Grohn em uso de terapia imunossupressora: relato de caso
}

\author{
Guilherme Gouvea Hollunder ${ }^{1}$ \\ Marcus Vinicius de Oliveira Ferreira ${ }^{2}$ \\ Caio Gomes Tabet ${ }^{2}$ \\ Valdeci Manoel de Oliveira ${ }^{2}$ \\ Jair Moreira Dias Junior ${ }^{2}$
}

${ }^{1}$ Faculdade de Medicina, Universidade Federal de Juiz de Fora, Brasil.

${ }^{2}$ Departamento de Ortopedia e Traumatologia, Hospital Universitário, Universidade Federal de Juiz de Fora, filial Empresa Brasileira de Serviços Hospitalares, Brasil.

\section{$\triangle$ Guilherme Hollunder}

Av. Presidente Itamar Franco, 1717, apt. 1503 , São Mateus, Juiz de Fora, Minas Gerais

CEP: $36016-321$

gulherme.hollunder@hotmail.com

\section{RESUMO}

Introdução: A infecção pelo Mycobacterium tuberculosis (Mt) é uma das dez principais causas de mortalidade no mundo. Apenas $0,5 \%$ dos casos de tuberculose (TB) se localizam na coluna, podendo ocorrer disfunções neurológicas, instabilidade mecânica e deformidades. O uso de drogas imunossupressoras para tratamento das doenças inflamatórias, como a doença de Crohn (DC), pode tornar o paciente suscetível a esta infecção oportunista. Objetivo: Relatar o caso de uma paciente com espondilite tuberculosa na vigência do tratamento de DC com terapia imunossupressora. Descrevemos seu tratamento clínico-cirúrgico, sua evolução e comparamos com a literatura vigente. Relato de Caso: Mulher, 51 anos, com diagnóstico de DC em uso contínuo de azatioprina iniciou quadro de dorsalgia, dificuldade de deambulação e perda ponderal sem repercussões neurológicas. Ressonância magnética (RM) e Tomografia Computadorizada (TC) da coluna dorsal apresentaram padrões sugestivos de espondilite tuberculosa. Em decorrência da evolução sem alívio da dor e com instabilidade vertebral iminente sob risco de fratura e lesão neurológica, foi indicada cirurgia para estabilização vertebral, descompressão de elementos neurais e coleta de material para estudo histopatológico. O resultado comprovou a presença de Mt na amostra. Paciente manteve internação por 28 dias com tratamento antibiótico, apresentando boa evolução, com melhora progressiva do quadro álgico e da deambulação. Durante toda a internação seu quadro de DC se manteve controlado. Conclusão: É importante considerar a espondilite tuberculosa como diagnóstico diferencial em pacientes com dorsalgia e sinais de alarme, especialmente, em vigência de tratamento imunossupressor, mesmo com PPD negativo.

Palavras-chave: Tuberculose; Doença de Crohn; Tuberculose da Coluna Vertebral; Fusão Vertebral.

\section{ABSTRACT}

Introduction: Infection with Mycobacterium tuberculosis (Mt) is one of the ten main causes of mortality in the world. Only $0.5 \%$ of tuberculosis (TB) cases are in the spine, leading to neurological disorders, mechanical instability and deformities. The use of immunosuppressive drugs to treat inflammatory diseases such as Crohn's disease (CD) can make the patient susceptible to this opportunistic infection. Objective: Report the case of a patient with tuberculous spondylitis undergoing treatment for CD with immunosuppressive therapy. We describe its clinical-surgical treatment and evolution comparing it with the current literature. Case Report: A 51-year-old woman, diagnosed with CD in continuous use of azathioprine, was admitted to our hospital for worsening middle back pain, difficulty in walking and weight loss without neurological repercussions. Magnetic Resonance (MRI) and Computerized Tomography (CT) of the dorsal column showed patterns suggestive of tuberculous spondylitis. Because of the disease's evolution without pain relief and associated with vertebral instability with imminent risk of fracture and neurological damage, surgery was indicated for vertebral stabilization, decompression of neural elements and removal of material for histopathological study. The result confirmed the presence of $\mathrm{Mt}$ in the sample. The patient was hospitalized for 28 days with antibiotic treatment, showing good evolution, with progressive improvement in pain and walking. During the entire hospital stay, her CD condition was kept under control. Conclusion: It is important to consider tuberculous spondylitis as a differential diagnosis in patients with back pain and alarm signs, especially in the presence of immunosuppressive treatment, even with negative PPD.

Key-words: Tuberculosis; Crohn's Disease; Spinal Tuberculosis; Spinal Fusion. 


\section{INTRODUÇÃO}

A infecção pelo Mycobacterium tuberculosis é uma das dez principais causas de mortalidade no mundo. ${ }^{1}$ A doença de Crohn está associada ao uso de drogas imunossupressoras, como a Azatioprina, tornando os pacientes suscetíveis a infecções oportunistas como a TB, possivelmente, transformando a forma latente desta doença em ativa por desequilibro da resposta imune. ${ }^{2-4}$

Nas infecções por M. tuberculosis, 90\% delas são pulmonares e apenas $10 \%$ em outras localizações, e destas, 5\% estão na coluna vertebral. Apesar de raro, ${ }^{5}$ o acometimento dessa região pode ser responsável por repercussões trágicas, como: déficits neurológicos, instabilidade mecânica e deformidade, motivados pela destruição dos corpos vertebrais e dos espaços intervertebrais. Além disso, mesmo quando o rastreio para TB for negativo, esta doença não deve ser esquecida. ${ }^{2,5-7}$ Ao se pesquisar nos bancos de dados científicos MEDLINE, PUBMED, SCIELO-BR e CAPES não foram encontrados relatos de caso de pacientes com doença de Crohn em uso contínuo de Azatioprina que evoluíram com manifestação de espondilite tuberculosa.

Assim, este trabalho objetiva relatar o caso de uma paciente portadora de DC em tratamento com imunossupressor que apresentou uma infecção oportunista com Mt, mesmo com exame de PPD negativo.

\section{RELATO DE EXPERIÊNCIA}

Paciente feminino, 51 anos, com diagnóstico de doença de Crohn (DC) desde 2004 em uso contínuo de imunossupressor - azatioprina $150 \mathrm{mg} / \mathrm{dia}$. Iniciou quadro de dorsalgia à direita, mecânica, pois piorava aos movimentos è deambulação. Apresentava dor à palpação da coluna vertebral na transição toracolombar e à flexão do tronco, associada a perda ponderal não intencional de $5 \mathrm{~kg}$ em 6 meses. Não apresentava irradiação da dor, alterações sensitivas ou motoras, anestesia em sela, disfunção esfincteriana, nem manobras de irritação radicular. Radiografia evidenciou perda de mais de $50 \%$ da altura do corpo de T11 e cifotização de 15 graus da coluna torácica devido a fratura patológica neste nível. Ressonância magnética (RM) de coluna dorsal (figura 1) apresentava coleções dentro dos corpos vertebrais e elementos posteriores de T10 e T11 - com extensão para partes moles, sugestiva de espondilite tuberculosa e/ ou espondilodiscite inespecífica (figura 2 ). Foi realizado teste pure protein derivative (PPD), cujo resultado foi não reator. Apresentava história de contato positiva, pois relatou que sua filha foi tratada de tuberculose (TB) dois anos antes do início dos seus sintomas e que o rastreio com radiografia torácica e o PPD também não

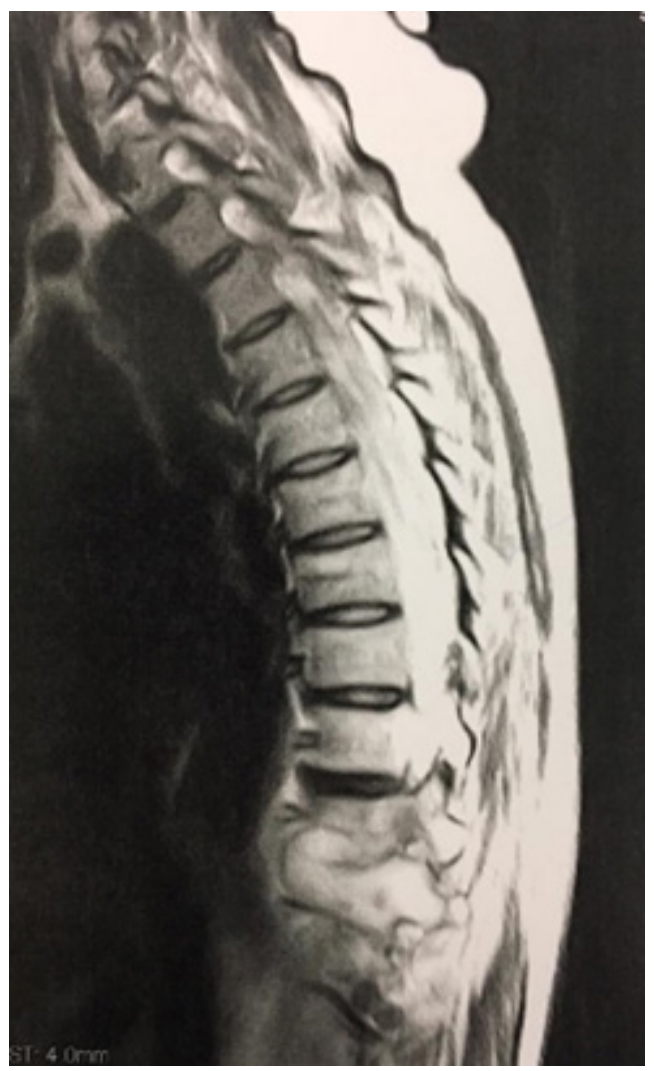

Figura 1: RM coluna torácica T2 corte parasagital à direita.
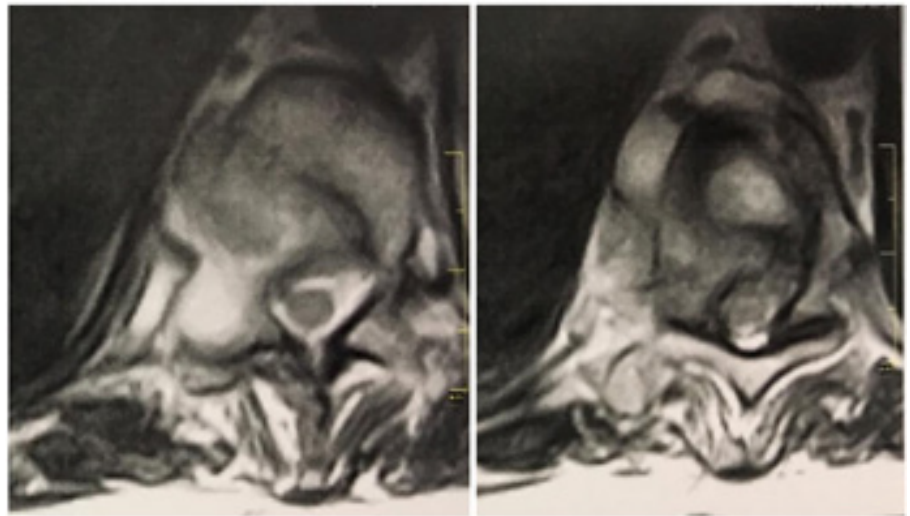

Figura 2: RM coluna torácica T2, axial dos segmentos $T 10$ e T11, respectivamente. 
apontaram alterações à época no exame da paciente.

Pelo exposto, suspendeu-se a Azatioprina para reestabelecimento da resposta imune. As suspeitas eram de metástase de tumor sólido com lesão osteolítica, mieloma múltiplo, espondilodiscite inespecífica ou tuberculose vertebral (TBV), mesmo com o exame negativo. Tomografia computadorizada (TC) da coluna torácica foi feita para melhor estudo da lesão e auxílio na programação do tratamento cirúrgico (figura 3). Foram identificadas alterações líticas destrutivas acometendo os corpos de T10 e determinando colapso de mais de $50 \%$ do corpo vertebral de T11, sinais indicativos de instabilidade mecânica, bem como redução da amplitude dos forames neurais de T10-T11 e rompimento das corticais ósseas com envolvimento de partes moles paravertebrais e epidural anterior de T10-T11. Foram encontradas também opacidades lineares de aspecto fibroelástico nas bases pulmonares. Os achados clínicos e de imagem direcionaram a conduta para cirurgia de descompressão, fixação, debridamento e coleta de material para análise laboratorial.

A paciente foi, então, submetida a artrodese toracolombar posterior sete níveis, em caráter eletivo. Foi encontrado grande quantidade de secreção purulenta em T11 (figura 4). Dois parafusos foram passados nos pedículos de T7, T8, T9, T12, L1 e L2 e hastes posicionadas lateralmente aos processos espinhosos com conexão de todo o sistema de implante (figura 5). Foi realizado descompressão ampla com laminectomia e costotrasversectomia esquerda. Utilizou-se fluoroscopia para garantir o bom posicionamento dos materiais implantados. Foi realizada coleta de material purulento para análise laboratorial e debridamento vigoroso. Os materiais encaminhados para cultura foram positivos aos testes de Bacilos Álcool-Ácido Resistentes (BAAR) e de reação de cadeira de polimerase (RCP) para TB.

Iniciou-se, então, terapia com rifampicina, isoniazida, pirazinamida e etambutol (RIPE) e foi mantida

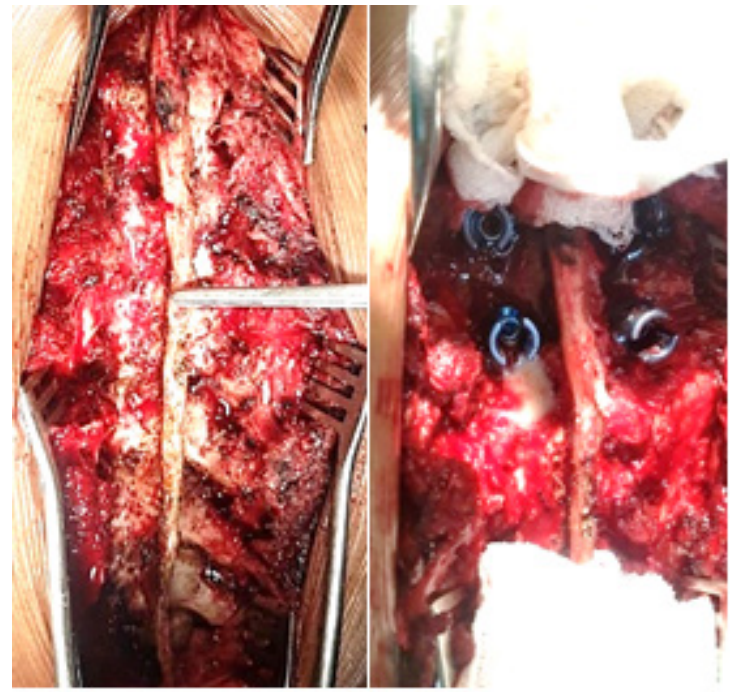

Figura 4: Secreção purulenta ao nível de T11 antes e após a

colocação de hastes e parafusos.

em tratamento hospitalar por 28 dias para tratamento com antibiótico. Durante a internação, a paciente apresentou exantema maculopapular pruriginoso após o início do RIPE que foi tratado com anti-histamínicos. A paciente evoluiu bem, com melhora progressiva do quadro álgico e da deambulação. Durante toda a internação seu quadro de DC se manteve controlado.

\section{DISCUSSÃO}

A DC é uma doença inflamatória crônica do trato gastrointestinal com recorrências e remissões frequentes. Resulta da associação entre susceptibilidade genética, fatores ambientais e alterações da microbiota intestinal que levam a perda da regulação das respostas imunes inata e adaptativa. ${ }^{9}$ Todos os segmentos

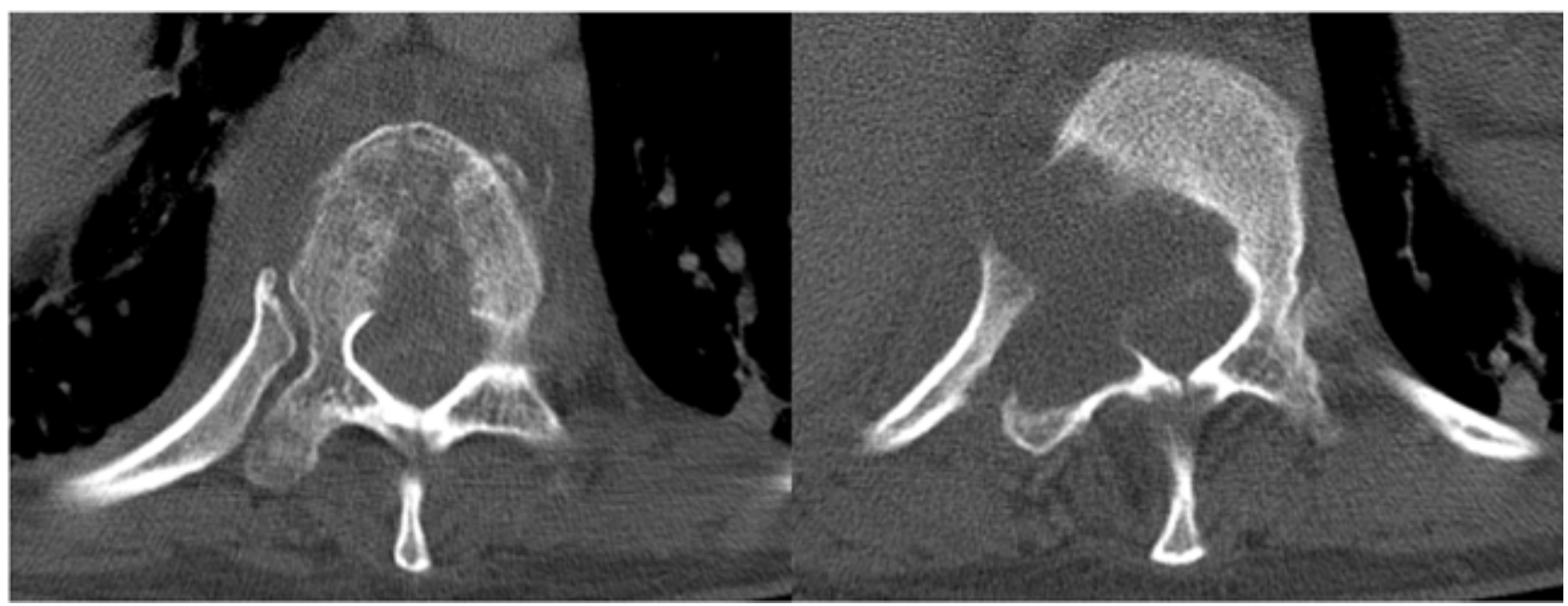

Figura 3: Tomografia computadorizada (corte axial) apresentando alterações líticas em vertebras T10 e T11, respectivamente. 


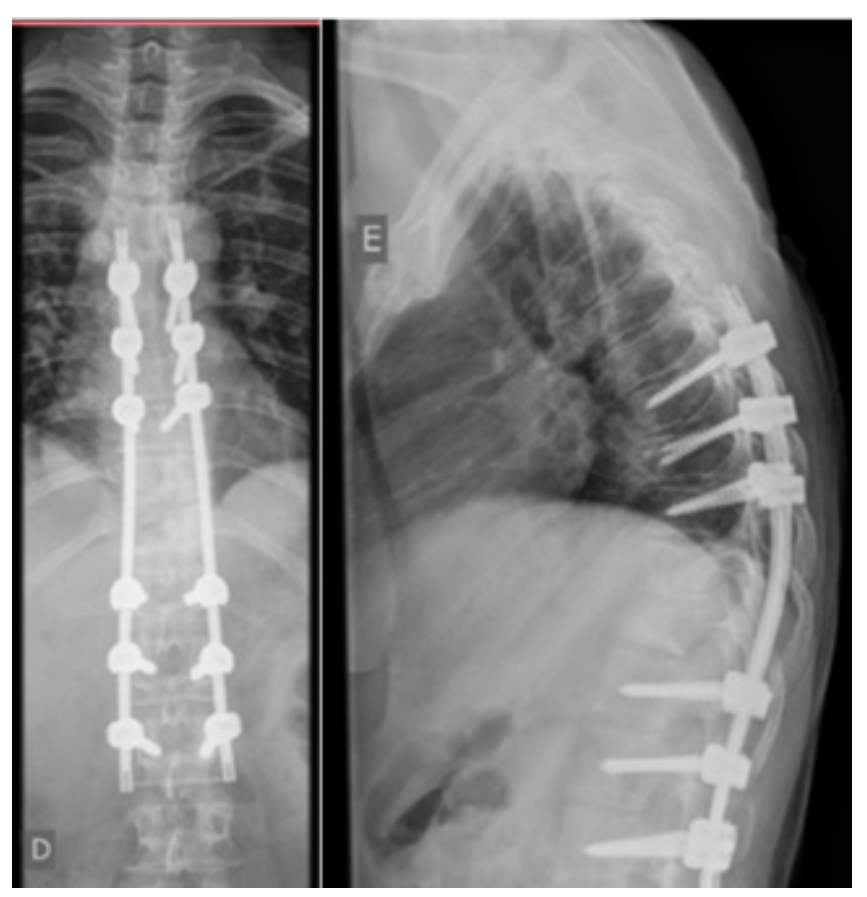

Figura 5: Radiografias do pós-operatório 1 ano em anteroposterior e perfil, demostrando bom alinhamento sagital.

do trato podem ser afetados, levando a formação de fístulas e abscessos, de caráter progressiva com disfunções e apresentações clínicas diversas. ${ }^{9}$ Seu tratamento envolve o uso de drogas imunossupressoras que favorecem infecções oportunistas como a TB em sua fase ativa. . $^{2,8}$

$O$ agente causador da TB, Mycobacterium tuberculosis, é encontrado em um terço da população mundial e em 2015 foi responsável por 10.4 milhões de casos de TB ativa e 1.8 milhões de óbitos mundo afora. ${ }^{1}$ Entre os indivíduos infectados, $10 \%$ desenvolvem a forma ativa da infecção e $90 \%$ se mantêm cronificados, fase caracterizada por uma adequada resposta imune contra o agente invasor que não permite que a doença evolua e, consequentemente, ausência da sintomatologia. ${ }^{1-3}$

Os sinais e sintomas da TB podem ser locais ou sistêmicos. A sintomatologia sistêmica inclui: perda de peso, anorexia, febre e sudorese noturna. A tuberculose vertebral (TBV) é uma forma de TB extrapulmonar que acomete mais frequentemente a transição da coluna lombar e torácica, levando a destruição dos corpos vertebrais e, posteriormente, dos discos intervertebrais. Dessa forma, pode levar a deformidade, instabilidade mecânica e déficits neurológicos. 5,6,12,13

A TB, em todas as suas manifestações, deve sempre ser levada em consideração em pacientes sob uso de tiopurinas, como a paciente do relato. ${ }^{2,10}$ Deve ser realizado rastreio por meio de PPD ou interferon gama release assays (IGRA) mesmo quando não existirem manifestações clínicas devido a possibilidade de TB em sua forma latente, ${ }^{2}$ por sua prevalência em $1 / 3$ da população mundial. ${ }^{1}$ Deve-se pesquisar, também, histórico de infecção ou contato com o agente etiológico e sinais na radiografia de tórax..$^{2,6,11}$ Quando TB ativa for diagnosticada, o protocolo RIPE deve ser iniciado imediatamente sendo mantido por 6 meses e os agentes imunossupressores descontinuados. ${ }^{1}$ Com a paciente, optou-se pela retirada da azatioprina para melhorar a resposta imunológica contra a infecção. Pode haver uso concomitante de drogas antituberculosas e imunossupressoras quando a DC está em sua fase aguda, no entanto, no relato não houveram manifestações de sintomas. ${ }^{2,9}$

Em uma revisão envolvendo mais de 2000 pacientes com a doença foi relatado lombalgia em $70 \%$, febre em $30 \%$ e déficits neurológicos em $23 \%$ a $76 \%$ dos pacientes avaliados. Os sintomas neurológicos incluem dor, parestesia e paresia, podendo haver evolução para paraplegia. Radiografias normalmente não apontam alterações nos estágios iniciais da doença, permitindo a visualização de acometimento ósseo quando $30 \%$ do corpo vertebral for comprometido. ${ }^{6}$ É possível observar aumento da cifose torácica, diminuição do espaço intervertebral e massas paravertebrais calcificadas. ${ }^{6,7}$ É valido destacar que o Mycobacterium tuberculosis não produz enzimas proteolíticas, o que implica diminuição do espaço dos discos vertebrais apenas em estágios mais avançados. 6 No relato, a radiografia da paciente apontava lesão em estágio avançado, apresentando perda de mais de $50 \%$ da altura de T11, diminuição do espaço intervertebral e cifose torácica. No entanto, não era possível visualizar as lesões líticas no restante dos segmentos vertebrais ou lesões de partes moles, sendo necessária a realização de TC ou RM.

A TC é mais bem utilizada para avaliação da extensão óssea, principalmente para o planejamento cirúrgico. ${ }^{6,7}$ Podem ser observadas precocemente massas paravertebrais e, tardiamente, fistulizações e calcificações das paredes dos abscessos. ${ }^{7}$ A RM, por sua vez, possui maior sensibilidade em relação às radiografias e maior especificidade em relação à TC no diagnóstico de TBV, permitindo visualização de compressão medular provocada por acúmulo de pus, alterações na medula óssea ou destruição discal. ${ }^{6}$ Devido ao maior detalhamento das imagens, permite a realização de diagnóstico diferencial com outras patologias, sendo portanto o exame diagnóstico de primeira linha para a TBV..$^{5-7}$

O diagnóstico definitivo da doença pode ser feito apenas pela cultura de material coletado por meio de biópsia ou aspiração da lesão. ${ }^{5-7}$ Técnicas laboratoriais utilizadas incluem detecção de produtos metabólicos - como o interferon gama, citocina liberada pelos linfócitos $\mathrm{T}$ quando expostos ao agente mesmo em infecções latentes - e RCP do M. tuberculosis, que amplifica o DNA da bactéria presente na amostra. ${ }^{6} \mathrm{~A}$ OMS sugere que todos pacientes que apresentam TB extrapulmonar devem realizar radiografias de tórax para investigação de TB pulmonar e receber tratamento com 
Hollunder et al. Espondilite tuberculosa no imunossuprimido por doença de Chron.

drogas antituberculosas. ${ }^{2,6}$

O tratamento cirúrgico é indicado quando o paciente apresenta deformidade espinhal, instabilidade mecânica e déficit neurológico que não respondem ao tratamento quimioterápico. Pode ser indicada, também, para descompressão de abscesso. ${ }^{5,6} \mathrm{~A}$ cifotização do segmento é a deformidade mais frequente na TBV que se desenvolve quando há acometimento da coluna torácica. ${ }^{6}$ Foi sugerido que cifoses maiores do que 30 graus em crianças deve ser corrigida cirurgicamente devido a possibilidade de problemas cardiorrespiratórios no futuro. ${ }^{14,15}$ Pacientes adultos são operados se apresentarem cifoses maiores do que 60 graus, mas deformidades menores podem ser operadas quando existir comorbidade importante. ${ }^{15}$ Dor que não responde ao tratamento é outra indicação pois muitas vezes está associada a deformidades e perda do alinhamento sagital. ${ }^{6}$

\section{CONCLUSÃO}

É importante considerar a espondilite tuberculosa como diagnóstico diferencial em pacientes com dorsalgia e sinais de alarme, especialmente, em vigência de tratamento imunossupressor, mesmo com PPD negativo, favorecendo o diagnóstico precoce desta doença prevalente e prevenindo graves lesões da coluna vertebral e da medula espinhal.

\section{CONFLITO DE INTERESSES}

Não há conflito de interesses.

\section{REFERÊNCIAS}

1. World Health Organisation. Global Health TB Report. 2018.

2. Inflammatory Bowel Disease Group, Chinese Society of Gastroenterology, Chinese Medical Association. Evidence-based consensus on opportunistic infections in inflammatory bowel disease. J Dig Dis. 2018; 19:54-65.

3. Riestra S, Francisco R, Arias-Guillén M, Saro C, GarcíaAlvarado $\mathrm{M}$ et al. Risk factors for tuberculosis in inflammatory bowel disease: anti-tumor necrosis factor and hospitalization. Rev Esp Enfermedades Dig. 2016; 108:541-9.

4. Toruner M, Loftus Jr EV, Harmsen ES, Zinsmeister AR, Orenstein $\mathrm{R}$ et al. risk factors for opportunistic infections in patients with inflammatory bowel disease. Gastroenterology. 2008; 134:929-36.

5. Dunn RN, Husien BM. Spinal tuberculosis review of current management. Bone Jt J. 2018; 100B:425-31.

6. Garg RK, Somvanshi DS. Spinal tuberculosis: a review. J
Spinal Cord Med. 2011; 34(5):440-54.

7. Chen $\mathrm{CH}$, Chen $\mathrm{YM}$, Lee CW, Chang YJ, Cheng $\mathrm{CY}$ et al. Early diagnosis of spinal tuberculosis. J Formos Med Assoc. 2016; 115(10):825-36.

8. Park DI, Hisamatsu T, Chen M, Ng SC, Ooi CJ et al. Asian Organization for Crohn's and Colitis and Asia Pacific Association of Gastroenterology consensus on tuberculosis infection in patients with inflammatory bowel disease receiving anti-tumor necrosis factor treatment. Part 1: risk assessment. Intest Res. 2018; 16(1):4-16.

9. Reyt V. Crohn's disease. Actual Pharm. 2018; 57:13-5.

10. Axelrad JE, Roy A, Lawlor G, Korelitz B, Lichtiger S. Thiopurines and inflammatory bowel disease: Current evidence and a historical perspective. World J Gastroenterol. 2016; 22(46):10103-17.

11. Bonovas S, Fiorino G, Allocca M, Lytras T, Nikolopoulos GK et al. Biologic therapies and risk of infection and malignancy in patients with inflammatory bowel disease: a systematic review and network meta-analysis. Clin Gastroenterol Hepatol. 2016; 14(10):1385-97.e10.

12. Kumar K. Spinal tuberculosis, natural history of disease, classifications and principles of management with historical perspective. Eur J Orthop Surg Traumatol. 2016; 26(6):551-8.

13. Dean A, Zyck S, Toshkezi G, Galgano M, Marawar S. Challenges in the diagnosis and management of spinal tuberculosis: case series. Cureus. 2019; 11(1):e3855.

14. Jain A, Dhammi I, Jain S, Mishra P. Kyphosis in spinal tuberculosis: prevention and correction. Indian J Orthop. 2010; $44: 127$.

15. Parthasarathy $R$, Sriram K, Santha T, Prabhakar R, Somasundaram PR et al. Short-course chemotherapy for tuberculosis of the spine: a comparison between ambulant treatment and radical surgery--ten-year report. J Bone Joint Surg Br. 1999; 81(3):464-71. 